\title{
A BOUNDED MOUNTAIN PASS LEMMA WITHOUT THE (PS) CONDITION AND APPLICATIONS
}

\author{
MARTIN SCHECHTER
}

\begin{abstract}
We present a version of the mountain pass lemma which does not require the (PS) condition. We apply this version to problems where the (PS) condition is not satisfied.
\end{abstract}

\section{INTRODUCTION}

The mountain pass lemma has been a very interesting tool in solving variational problems (cf., e.g., $[4,6,7,8,9,10])$. It concerns a real-valued $C^{\prime}$ functional $G(u)$ defined on a real Banach space $X$ for which one desires to find a critical point, i.e., a point where $G^{\prime}(u)=0$. In the simplest version one finds two points $e_{1}, e_{2}$ which are separated by a set $M$ such that for some number $a$

$$
G\left(e_{i}\right)<a, \quad G(u) \geq a, \quad u \in M .
$$

This resembles the situation of a traveler trying to cross a mountain range without climbing higher than necessary. If we can find a continuous path connecting the two points which does not take the traveler higher than any other such path, it is expected that this path will produce a critical point.

However, there is a difficulty which must be addressed. One must allow the competing paths to roam freely, and conceivably they can take the traveler to infinity while he is trying to cross some local mountains. For the mathematician this can make it extremely difficult for him to locate critical points. To deal with this problem most researchers use the Palais-Smale (PS) condition which requires the sequences $\left\{u_{k}\right\}$ satisfying $\left|G\left(u_{k}\right)\right| \leq C, G^{\prime}\left(u_{k}\right) \rightarrow 0$ to have convergent subsequences. This has the effect of allowing one to deal with unbounded regions in a uniform way. However, there are many problems for which the (PS) condition is not satisfied. One approach is to require (PS) on bounded regions and control the growth of $\left\|G^{\prime}(u)\right\|^{-1}$ near infinity (cf. $\left.[9,7]\right)$. This has the same effect in that it allows one to deal with unbounded regions in a uniform way.

In this paper we consider some problems that do not yield to either approach. Our method is to restrict the competing paths to a bounded region. This can be done only if one can be assured that the paths will not leave the region as they approach the optimal one. We accomplish this by imposing a boundary

Received by the editors February 10, 1989 and, in revised form, March 8, 1990.

1980 Mathematics Subject Classification (1985 Revision). Primary 35J65, 47H15, 58E05, 49B27.

Research supported in part by NSF Grant. 
condition. For the cases considered here we take the region to be the ball $\|u\| \leq R$ and require that there be a $\Theta<1$ such that

$$
G^{\prime}(u) u \geq-\Theta\left\|G^{\prime}(u)\right\|\|u\|
$$

whenever $u$ satisfies

$$
c-\sigma \leq G(u) \leq c+\sigma, \quad\|u\|=R .
$$

(The constants $c$ and $\sigma$ depend on the problem.) If (1.1) holds for such $u$, we will be guaranteed that competing paths will remain in the ball if they are close to the optimal path. In order to apply (1.1) we were required to generalize the concept of pseudogradient (cf., e.g., [2, 3]). We need a mapping $Y(u)$, locally Lipschitz, such that for some $\alpha>0$

$$
\|Y(u)\| \leq 1, \quad G^{\prime}(u) Y(u) \geq \alpha\left\|G^{\prime}(u)\right\|, \quad u \in X,
$$

and

$$
(Y(u), u)>0, \quad\|u\|=R, \quad u \text { satisfies (1.2). }
$$

(A Hilbert space framework is used.) We prove this under assumption (1.1) provided $\alpha<1-\Theta$. It is property (1.4) that keeps the competing paths inside the ball $\|u\| \leq R$.

As an application for which the (PS) condition does not apply, we have

Theorem 1. Let $\Omega$ be a smooth bounded domain in $\mathbf{R}^{n}$, and let $f(x, t)$ be a Carathéodory function satisfying $|f(x, t)| \leq V_{1}(x),|t| \leq 1, V_{1}(x) \in L^{1}(\Omega)$, and

$$
F(x, t):=\int_{0}^{t} f(x, s) d s \leq \frac{1}{2} b(x) t^{2}+W(x), \quad t \in \mathbf{R},
$$

where $b(x) \leq b:=\lambda_{l+1}-\lambda_{l}, W(x) \geq 0$ is in $L^{1}(\Omega)$. Here $\lambda_{l}$ is an eigenvalue of the Dirichlet problem

$$
-\Delta u=\lambda u \quad \text { in } \Omega, \quad u=0 \text { on } \partial \Omega,
$$

with eigenspace $E_{l}$, and $\lambda_{l+1}$ is the next larger eigenvalue. Let

$$
H(x, t):=F(x, t)-\frac{1}{2} t f(x, t),
$$

and assume that $|H(x, t)| \leq W_{1}(x) \in L^{1}(\Omega)$ and

$$
H(x, t) \rightarrow H_{ \pm}(x) \text { as } t \rightarrow \pm \infty \text { a.e. }
$$

with

$$
\int_{v>0} H_{+}(x) d x+\int_{v<0} H_{-}(x) d x \geq B:=\int W(x) d x,
$$

for all solutions $v \not \equiv 0$ of

$$
-\Delta u-\lambda_{l} u=b_{+} u_{+}-b_{-} u_{-} \quad \text { in } \Omega, \quad u=0 \quad \text { on } \partial \Omega,
$$

where

$$
b_{ \pm}(x):=\limsup _{t \rightarrow \pm \infty} 2 t^{-2} F(x, t) \geq 0
$$

and

$$
u_{+}(x)=\max [u(x), 0], \quad u_{-}(x)=u_{+}(x)-u(x) .
$$


Finally, assume that there does not exist a $w \in E_{l+1} \backslash\{0\}$ such that

$$
\begin{array}{lll}
b_{+}(x) \equiv b, & H_{+}(x) \equiv W(x) & \text { when } w>0, \\
b_{-}(x) \equiv b, & H_{-}(x) \equiv W(x) & \text { when } w<0 .
\end{array}
$$

Then the nonlinear Dirichlet problem

$$
-\Delta u-\lambda_{l} u=f(x, u) \quad \text { in } \Omega, \quad u=0 \quad \text { on } \partial \Omega,
$$

has a solution.

Another application is

Theorem 2. Let $\Omega$ be a smooth bounded domain in $\mathbf{R}^{n}$ and let $f(x, t)$ be a Carathéodory function such that

$$
|f(x, t)| \leq C|t|^{\gamma}+k_{1}(x), \quad t \in \mathbf{R},
$$

with $1 \leq q=1+\gamma<2, k_{1} \in L^{q^{\prime}}(\Omega)$. Let $\lambda_{l}$ be an eigenvalue of the problem (1.6) with eigenspace $E_{l}$. Assume that

$$
|t|^{-\gamma} f(x, t) \rightarrow h_{ \pm}(x) \text { a.e. as } t \rightarrow \pm \infty,
$$

and

$$
B(v) B(-v)>0, \quad v \in E_{l} \backslash\{0\},
$$

where

$$
B(v)=\int_{v>0} h_{+}(x)|v(x)|^{q} d x-\int_{v<0} h_{-}(x)|v(x)|^{q} d x .
$$

Then the problem (1.12) has a solution.

Remark 1 . One might be tempted to replace (1.14) by the seemingly weaker assumption

$$
B(v) \neq-\mu B(-v), \quad v \in E_{l} \backslash\{0\}, 0 \leq \mu \leq 1 .
$$

We shall show in $\S 4$ that this implies (1.14).

Remark 2. Hypothesis (1.14) implies that either

$$
B(v)>0, \quad v \in E_{l} \backslash\{0\},
$$

or

$$
B(v)<0, \quad v \in E_{l} \backslash\{0\} .
$$

That is, $B(v)$ does not change sign on $E_{l}$. For $B(v)$ is a continuous function of $v$ on $E_{l}$, and if $B\left(v_{1}\right)>0, B\left(v_{2}\right)<0$, then $(1-\theta) v_{1}+\theta v_{2} \neq 0$ for $0<\theta<1$. Otherwise there would be a $\theta$ such that $v_{1}=-\theta v_{2} /(1-\theta)$. By $(1.14)$

$$
B\left(v_{1}\right) B\left(\theta v_{2} /(1-\theta)\right)>0 .
$$

Consequently

$$
\left(\theta^{q} /(1-\theta)^{q}\right) B\left(v_{1}\right)<0,
$$

a contradiction. But $B\left([1-\theta] v_{1}+\theta v_{2}\right)$ is positive for $\theta=0$ and negative for $\theta=1$. This would imply that $B(v)=0$ for some $v \neq 0$.

As another application we have 
Theorem 3. Let $\Omega$ be a bounded smooth domain in $\mathbf{R}^{n}$, and let $\lambda_{l}, b$ be as above. Let $f(x, t)$ be a Carathéodory function such that

$$
\begin{gathered}
-W_{1}(x) \leq F(x, t) \leq \frac{1}{2} b t^{2}+W(x), \\
|f(x, t)| \leq V(x) \in L^{2}(\Omega), \quad t f(x, t) \leq W_{2}(x),
\end{gathered}
$$

where $W, W_{1}, W_{2}$ are in $L^{1}(\Omega)$. Assume also that

$$
F(x, t) \rightarrow F_{ \pm}(x) \text { a.e. as } t \rightarrow \pm \infty \text {, }
$$

and

$$
\int_{v>0} F_{+}(x) d x+\int_{v<0} F_{-}(x) d x>B:=\int W(x) d x, \quad v \in E_{l} \backslash\{0\} .
$$

Then the problem (1.12) has a solution.

The roots of the mountain pass lemma go back to the "method of steepest descent", the "deformation theorem" and the "minimax principle" (cf. [1-3]). It was formulated in the present context by Ambrosetti and Rabinowitz [4] basing it on a proof by Clark [5]. Since then there have been several generalizations (cf. [6] for a survey). To the best of the author's knowledge, they all use either the (PS) condition or an estimate of the growth of $\left\|G^{\prime}(u)\right\|^{-1}$ near infinity.

Some of our applications have been considered by others using different methods. In particular we mention Landesman and Lazer [1], De Figueiredo [14], Gaines and Mawhin [15], Tarafdar [16].

Our mountain pass lemma is stated and proved in $\S 3$. Applications are stated in $\S \S 1$ and 2 and proved in $\S 4$.

We give two more applications of the method. Let

$$
f(x, t)=f_{+}(x) t_{+}-f_{-}(x) t_{-}+W(x) \psi^{\prime}(t),
$$

where $W(x) \geq 0$ is a function in $L^{1}(\Omega)$ and $\psi(t) \in C^{1}(\mathbf{R})$ satisfies

$$
\begin{gathered}
|\psi(t)|+\left|\psi^{\prime}(t)\right| \leq C, \quad \psi(t)<1, \quad \psi(0)=0, \\
\varphi(t):=\psi-\frac{1}{2} t \psi^{\prime} \rightarrow 1 \quad \text { as }|t| \rightarrow \infty .
\end{gathered}
$$

We let $\lambda_{l}$ be an eigenvalue of the Dirichlet problem (1.6) with eigenspace $E_{l}$. We have

Theorem 4. Assume in addition that $0 \leq f_{ \pm}(x) \leq \lambda_{l+1}-\lambda_{l}$, and that

$$
\int_{v>0} f_{+}(x) v(x)^{2} d x+\int_{v<0} f_{-}(x) v(x)^{2} d x>0, \quad v \in E_{l} \backslash\{0\} .
$$

Then (1.12) has a solution.

In our next theorem we do not require (1.25) to hold on the whole of $E_{l}$. We can allow $E_{l}$ to be split up into the direct sum of two orthogonal subspaces with (1.25) holding on one of them and the opposite inequality holding on the other. We can do this in the following way. Let $E_{l}=N_{0} \oplus N_{1}$, where the $N_{i}$ are orthogonal subspaces. Let

$$
N^{\prime}=\bigoplus_{j<l} E_{j}, \quad N=N^{\prime} \oplus N_{0} .
$$

We have 
Theorem 5. Under hypotheses (1.22)-(1.24) assume that $\lambda_{l-1}-\lambda_{l} \leq f_{ \pm}(x) \leq$ $\lambda_{l+1}-\lambda_{l}$ and that

(1.26) $-\left(\left[\Delta+\lambda_{l}\right] v, v\right)<\int_{v>0} f_{+}(x) v(x)^{2}+\int_{v<0} f_{-}(x) v(x)^{2}, \quad v \in N \backslash\{0\}$,

and

$$
-\left(\left[\Delta+\lambda_{l}\right] w, w\right) \geq \int_{w>0} f_{+}(x) w(x)^{2}+\int_{w<0} f_{-}(x) w(x)^{2}, \quad w \perp N .
$$

Then (1.12) has a solution.

I would like to thank Louis Nirenberg for interesting conversations.

\section{Semilinear eQuations}

Let $A$ be a selfadjoint operator on $L^{2}(\Omega)$, and let $f(x, t)$ be a function from $\Omega \times \mathbf{R}$ to $\mathbf{R}$. We are concerned with finding solutions to the equation

$$
A u=f(x, u), \quad u \in D(A) .
$$

A function $u \in D:=D\left(|A|^{1 / 2}\right)$ will be called a semistrong solution of $(2.1)$ if

$$
2 a(u, v)=(f(x, u), v), \quad v \in D,
$$

where

$$
a(u, v)=\frac{1}{2}(A u, v), \quad a(u)=a(u, u),
$$

and

$$
(u, v)=\int_{\Omega} u(x) v(x) d x, \quad\|u\|^{2}=(u, u) .
$$

It is clear that any semistrong solution $u$ for which $f(x, u)$ is in $L^{2}(\Omega)$ is an actual (strong) solution. We call a semistrong solution a solution.

We make the following assumptions.

I. The essential spectrum $\sigma_{e}(A)$ of $A$, if any, is positive. Thus any nonpositive point of the spectrum $\sigma(A)$ of $A$ is an isolated eigenvalue of finite multiplicity.

II. We assume that $f(x, t)$ is a Carathéodory function. This means that it is measurable in $x$ for every $t$ and continuous in $t$ for almost every $x$. Also for some $q \geq 1$ such that

$$
\|h\|_{q}^{2} \leq C_{1} a(h)+C_{2}\|h\|^{2}, \quad h \in D,
$$

we assume that

$$
|f(x, t)| \leq V_{0}(x)^{q}|t|^{q-1}+V_{0}(x) k_{1}(x),
$$

where $V_{0}(x)>0$ is a compact operator from $D$ to $L^{q}(\Omega)$ and $k_{1}$ is in $L^{q^{\prime}}(\Omega)$. Here $q^{\prime}:=q /(q-1)$ and

$$
\|h\|_{q}:=\left(\int_{\Omega}|h(x)|^{q} d x\right)^{1 / q} .
$$

III. We assume that there is a subspace $N_{0}$ of $N(A)$ (possibly empty or the whole of $N(A))$ such that

$$
\begin{gathered}
c_{1}:=\inf _{M} G>-\infty, \\
c_{0}:=\lim \sup \{G(v),\|v\| \rightarrow \infty, v \in N\}<c_{1},
\end{gathered}
$$


where

$$
\begin{gathered}
N:=N^{\prime} \oplus N_{0}, \quad N^{\prime}:=\bigoplus_{\lambda<0} N(A-\lambda), \quad M:=D \cap N^{\perp}, \\
G(u):=a(u)-\int_{\Omega} F(x, u) d x, \\
F(x, t):=\int_{0}^{t} f(x, s) d s .
\end{gathered}
$$

IV. For each $c \geq c_{1}$ for which the set $c \leq G(u) \leq c+1$ is unbounded, there is a $\Theta<1$ such that

$$
b(c):=\liminf _{\substack{\|u\| \rightarrow \infty \\ c \leq G(u) \leq c+1}}\left\{\int_{\Omega} H(x, u) d x+\frac{\Theta}{2}\left\|G^{\prime}(u)\right\|\|u\|\right\}>-c,
$$

where

$$
H(x, t):=F(x, t)-\frac{1}{2} t f(x, t)
$$

Theorem 6. Under hypotheses I-IV, equation (2.1) has a solution.

Theorem 7. Hypothesis IV can be replaced by

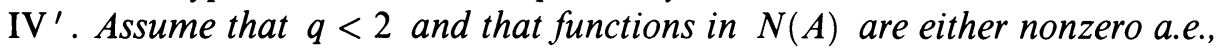
or vanish identically. Assume also that there are functions $W_{0}(x), W_{1}(x)$ in $L^{1}(\Omega)$ such that

$$
\begin{gathered}
\liminf _{|t| \rightarrow \infty} H(x, t) \geq W_{0}(x) \quad \text { a.e., } \\
H(x, t) \geq W_{1}(x) \quad \text { a.e., } \quad t \in \mathbf{R},
\end{gathered}
$$

and

$$
b_{0}+c_{1}>0
$$

where

$$
b_{i}:=\int_{\Omega} W_{i}(x) d x, \quad i=0,1,
$$

and $H(x, t)$ is given by (2.9).

Proposition 1. Hypothesis III will be fulfilled if $N_{0}=N(A)$,

$$
F(x, t)<\frac{1}{2} \lambda_{+} t^{2}+W_{0}(x), \quad t \in \mathbf{R},
$$

and

$$
\liminf _{|t| \rightarrow \infty} F(x, t) \geq W_{2}(x), \quad F(x, t) \geq W_{3}(x) \quad \text { a.e., }
$$

where $\lambda_{+}$is the smallest positive point in $\sigma(A)$, the $W_{i}(x)$ are in $L^{1}(\Omega)$ with

$$
b_{2}:=\int_{\Omega} W_{2}(x) d x>-b_{0}:=-\int_{\Omega} W_{0}(x) d x,
$$

and functions in $N$ which vanish on a set of positive measure vanish identically. 
Theorem 8. If there is a function $V_{1}(x)$ in $L^{2}(\Omega)$ such that

$$
|f(x, t)| \leq V_{1}(x) \text { a.e., } \quad t \in \mathbf{R},
$$

then hypothesis IV can be replaced by

IV". Functions in $N(A)$ not identically zero are nonzero a.e. There is a real number $\Theta,|\Theta|<1$, and functions $W_{4}(x), W_{5}(x)$ in $L^{1}(\Omega)$ such that

$$
\liminf _{|t| \rightarrow \infty} H_{\Theta}(x, t) \geq W_{4}(x), \quad H_{\Theta}(x, t) \geq W_{5}(x) \quad \text { a.e. }
$$

and

$$
b_{4}+c_{1}>0
$$

where

$$
H_{\Theta}(x, t):=F(x, t)-\frac{1}{2}(1+\Theta) t f(x, t),
$$

and

$$
b_{i}:=\int_{\Omega} W_{i}(x) d x .
$$

Theorem 9. If $f(x, t)$ satisfies (2.17) for some $V_{1} \in L^{2}(\Omega)$, then (2.4) and (2.5) are implied by

$$
\int_{\Omega} F(x, v) d x \rightarrow \infty \quad \text { as }\|v\| \rightarrow \infty, \quad v \in N_{0},
$$

and

$$
\alpha_{1}:=\sup _{N_{1}} \int_{\Omega} F(x, v) d x<\infty,
$$

where $N(A)=N_{0} \oplus N_{1}$.

\section{The mountain pass without the Palais-Smale Condition}

In this section we prove a version of the mountain pass lemma in which the competing curves or surfaces are restricted to a bounded region. We do this by imposing "boundary conditions". In order to obtain the most general boundary conditions, we generalize the notion of pseudogradient (cf. [3]). For this purpose we have:

Theorem 10. Let $\mathscr{H}$ be a Hilbert space, and let $X(u)$ be a continuous mapping of $\mathscr{H}$ into itself such that $X(u) \neq 0$ for all $u$. Let $v_{i}(u)$ be continuous mappings such that $v_{i}(u)$ does not vanish on a closed set $Q_{i}$. Assume that

$$
\left(v_{i}(u), v_{j}(u)\right)=0, \quad i \neq j,
$$

and that there are numbers $\Theta_{i} \geq 0$ such that

$$
\Theta^{2}=\sum \Theta_{i}^{2}<1
$$

and

$$
\left(X(u), v_{i}(u)\right) \leq \Theta_{i}\|X(u)\|\left\|v_{i}(u)\right\|, \quad u \in Q_{i}
$$


If $\alpha<1-\Theta$, then there is a locally Lipschitz map $Y(u)$ such that $\|Y(u)\| \leq 1$ and

$$
\begin{gathered}
(X(u), Y(u)) \geq \alpha\|X(u)\|, \quad u \in \mathscr{H}, \\
\left(Y(u), v_{i}(u)\right)<0, \quad u \in Q_{i} .
\end{gathered}
$$

We shall give the proof of Theorem 10 at the end of this section. Now we shall use it in proving

Theorem 11. Let $G(u) \in C^{\prime}(\mathscr{H}, \mathbf{R})$ satisfy

$$
\begin{gathered}
u_{k} \rightarrow u \quad \text { weakly, } \quad\left|G\left(u_{k}\right)\right| \leq C, \\
G^{\prime}\left(u_{k}\right) \rightarrow 0 \text { imply that } G^{\prime}(u)=0 .
\end{gathered}
$$

assume that $\mathscr{H}=N \oplus M$, where $N, M$ are orthogonal subspaces with $\operatorname{dim} N<$ $\infty$. Assume that there are constants $R \geq R_{0}>0$ such that

$$
c_{0}:=\max _{\partial B_{0}} G<c_{1}:=\inf _{B} G \leq c_{2}:=\max _{B_{0}} G,
$$

where

$$
B_{0}:=\left\{v \in N \mid\|v\| \leq R_{0}\right\}, \quad B:=\{w \in M \mid\|w\| \leq R\},
$$

and that for each $c$ satisfying $c_{1} \leq c \leq c_{2}$ there are constants $\sigma>0, \Theta<1$ such that

$$
\left(G^{\prime}(u), u\right) \geq-\Theta R\left\|G^{\prime}(u)\right\|,
$$

holds for all $u$ satisfying

$$
c-\sigma \leq G(u) \leq c+\sigma, \quad\|u\|=R .
$$

Then there is a $u \in \mathscr{H}$ satisfying $G^{\prime}(u)=0$.

Proof. Let $Q$ be the ball $\|u\| \leq R$, and assume that $G^{\prime}(u) \neq 0$ for all $u$. Let $\mathscr{S}$ denote the set of all continuous mappings $\varphi(v)$ of $B_{0}$ into $Q$ such that

$$
\varphi(v)=v, \quad v \in \partial B_{0} .
$$

It is clear that

$$
\varphi\left(B_{0}\right) \cap B \neq \phi, \quad \varphi \in \mathscr{S} .
$$

Let

$$
c=\inf _{\varphi \in \mathscr{S}} \sup _{v \in B_{0}} G(\varphi(v)) .
$$

Then $c_{1} \leq c \leq c_{2}$, and there exist constants $\sigma>0, \Theta<1$ such that (3.9) holds for all $u$ satisfying (3.10). Let $\varepsilon>0$ be such that $3 \varepsilon<c_{1}-c_{0}$ and $3 \varepsilon<\sigma$. Let

$$
\begin{aligned}
& Q_{1}=\{u \in Q \mid c-\varepsilon \leq G(u) \leq c+\varepsilon\}, \\
& Q_{2}=\{u \in Q \mid G(u) \leq c-2 \varepsilon \text { or } G(u) \geq c+2 \varepsilon\} .
\end{aligned}
$$

There is a constant $a>0$ such that $\left\|G^{\prime}(u)\right\| \geq a$ for all $u \in Q$ satisfying (3.10). For otherwise there would be a sequence $\left\{u_{k}\right\}$ of such elements such that $G^{\prime}\left(u_{k}\right) \rightarrow 0$. A subsequence would converge weakly to an element $u \in Q$, and (3.6) would imply that $G^{\prime}(u)=0$. Let

$$
\eta(u)=d\left(u, Q_{2}\right) /\left[d\left(u, Q_{1}\right)+d\left(u, Q_{2}\right)\right] .
$$


Then $\eta$ is a Lipschitz continuous function which equals 1 on $Q_{1}$, vanishes on $Q_{2}$ and satisfies

$$
\eta(u) \neq 0 \quad \text { for } u \notin Q_{2}, \quad 0 \leq \eta(u) \leq 1 .
$$

By Theorem 10 there is a locally Lipschitz continuous mapping $Y(u)$ such that

$$
\left(G^{\prime}(u), Y(u)\right) \geq \alpha\left\|G^{\prime}(u)\right\|, \quad\|Y(u)\| \leq 1, \quad u \in \mathscr{H},
$$

and

$$
(Y(u), u)>0, \quad u \text { satisfies }(3.10),
$$

where $\alpha<1-\Theta$. (Here there is only one $v_{i}(u)$, which we take to be $-u$. $Q_{i}$ is taken as the set of those $u$ satisfying (3.10).) By (3.13) there is a $\varphi \in \mathscr{S}$ such that

$$
G(\varphi(v))<c+\varepsilon, \quad v \in B_{0}
$$

We let $\rho(t)=\rho(t, v)$ be the solution of

$$
\begin{gathered}
d \rho / d t=-\eta(\rho(t)) Y(\rho(t)) /\|Y(\rho(t))\|, \\
\rho(0)=\varphi(v) .
\end{gathered}
$$

Since $Y(u)$ is Lipschitz continuous and does not vanish, $\rho(t, v)$ exists for all $t>0$ and $v \in B_{0}$. Note that $\partial B_{0} \subset Q_{2}$. Then $\rho(t, v)=v$ for all $v \in \partial B_{0}$. We claim that $\rho(t)$ never leaves $Q . \rho(t)$ cannot leave via a point which is in $Q_{2}$ because $\eta=0$ in $Q_{2}$. Moreover, $\rho(t)$ cannot approach points of $\partial Q$ which are not in $Q_{2}$ because $\eta>0$ at such points and $\rho(t)$ is directed inward at them and consequently in the neighborhood of such points by (3.15) and (3.18). Now

$$
d\|\rho(t)-\varphi(v)\| / d t \leq\|d \rho / d t\| \leq 1,
$$

and consequently

$$
\|\rho(t)-\varphi(v)\| \leq t
$$

Moreover

$$
\begin{aligned}
d(G(\rho(t)) / d t & =-\eta(\rho(t)) G^{\prime}(\rho(t)) Y(\rho(t)) /\|Y(\rho(t))\| \\
& \leq-\eta(\rho(t)) \alpha\left\|G^{\prime}(\rho(t))\right\| .
\end{aligned}
$$

Thus we have

$$
G\left(\rho\left(t_{2}\right)\right) \leq G\left(\rho\left(t_{1}\right)\right) \leq G(\varphi(v)), \quad t_{1}<t_{2} .
$$

Let $T$ satisfy $2 \varepsilon<\alpha a T$. If $\rho(t)$ does not leave $Q_{1}$ for $0 \leq t \leq T$, then

$$
\begin{aligned}
G(\rho(T))-G(\varphi(v)) & \leq-\alpha \int_{0}^{T}\left\|G^{\prime}(\rho(t))\right\| d t \\
& \leq-\alpha a T<-2 \varepsilon .
\end{aligned}
$$

On the other hand, if there is a $t_{1}$ such that $0 \leq t_{1} \leq T$ and $\rho\left(t_{1}\right)$ is not in $Q_{1}$, then we must have

$$
G\left(\rho\left(t_{1}\right)\right)<c-\varepsilon
$$

since we cannot have $G\left(\rho\left(t_{1}\right)\right)<c+\varepsilon$. Thus

$$
G(\rho(T)) \leq G\left(\rho\left(t_{1}\right)\right)<c-\varepsilon .
$$


Let $\varphi_{1}(v)=\rho(T, v)$. Since $\rho(t)$ never leaves $Q$, we see that $\varphi_{1}$ is in $\mathscr{S}$. Moreover, (3.23) and (3.24) show that

$$
G\left(\varphi_{1}(v)\right)<c-\varepsilon, \quad v \in B_{0} .
$$

This contradicts (3.13), showing that the assumption $G^{\prime}(u) \neq 0$ is incorrect.

In proving Theorem 10 we shall make use of

Lemma 1. Let $\Theta_{i} \geq 0$ be such that

$$
\Theta^{2}=\sum \Theta_{i}^{2}<1,
$$

and let $\alpha>0$ satisfy $\alpha<1-\Theta$. Then for any elements $u \neq 0, v_{i} \neq 0$, such that

$$
\left(u, v_{i}\right) \leq \Theta_{i}\|u\|\left\|v_{i}\right\|, \quad\left(v_{i}, v_{j}\right)=0, \quad i \neq j,
$$

there is an element $h$ such that

$$
(u, h) \geq \alpha\|u\|\|h\|, \quad\left(h, v_{i}\right)<0 \text { for each } i .
$$

Proof. We may assume that $u$ and the $v_{i}$ are unit vectors. We take $h$ of the form

$$
h=u-\sum \beta_{i} v_{i}, \quad \beta_{i} \geq 0 .
$$

If $\beta^{2}=\sum \beta_{i}^{2}$, then

$$
\|h\| \leq 1+\beta, \quad\left(h, v_{i}\right) \leq \Theta_{i}-\beta_{i},
$$

and

$$
(u, h) \geq 1-\sum \beta_{i} \Theta_{i} \geq 1-\beta \Theta .
$$

If we take $\beta_{i}>\Theta_{i}$ such that $\alpha(1+\beta) \leq 1-\beta \Theta$, (3.27) will be satisfied. This can be done because of the assumptions on $\Theta$ and $\alpha$.

Proof of Theorem 10. Let $\alpha^{\prime}$ be any number satisfying $\alpha<\alpha^{\prime}<1-\Theta$. By Lemma 1 for each $u$ there is an element $h(u)$ such that

$$
\begin{aligned}
(X(u), h(u)) & \geq \alpha^{\prime}\|X(u)\|, \quad\|h(u)\|=1, \quad u \in \mathscr{H}, \\
\left(h(u), v_{i}(u)\right)<0, \quad u \in Q_{i} . &
\end{aligned}
$$

(If $u$ is not in $Q_{i}$, (3.3) does not hold and (3.29) is not needed. We merely take $\beta_{i}=0$ in the construction of $h$ in Lemma 1.) By the continuity of $X(u)$ and the $v_{i}(u)$, there is a neighborhood $N(u)$ of each $u$ such that

$$
\begin{aligned}
& (X(g), h(u)) \geq \alpha\|X(g)\|, \quad g \in N(u), \\
& \left(h(u), v_{i}(g)\right)<0, \quad u \in Q_{i}, g \in N(u) .
\end{aligned}
$$

If $u$ is not in $Q_{i}$, we require that $N(u)$ not intersect $Q_{i}$. The set of all such neighborhoods covers $\mathscr{H}$. There is a locally finite, locally Lipschitz partition of unity $\left\{\psi_{\tau}\right\}$ subordinate to a refinement of this covering (cf., e.g., [3]). Let

$$
Y(g)=\sum \psi_{\tau}(g) h_{\tau}\left(u_{\tau}\right)
$$

$Y(g)$ is locally Lipschitz continuous since $u_{\tau}$ is constant on the support of $\psi_{\tau}$. Now

$$
\|Y(g)\| \leq \sum \psi_{\tau}(g)\left\|h_{\tau}\right\| \leq 1,
$$


and for $g \in Q_{i}$

$$
\left(Y(g), v_{i}(g)\right)=\sum \psi_{\tau}(g)\left(h_{\tau}\left(u_{\tau}\right), v_{i}(g)\right)<0 .
$$

Also for any $g$

$$
(X(g), Y(g))=\sum \psi_{\tau}(g)\left(X(g), h_{\tau}\left(u_{\tau}\right)\right) \geq \sum \psi_{\tau}(g) \alpha\|X(g)\|=\alpha\|X(g)\| .
$$

Thus (3.4) and (3.5) hold.

\section{THE REDUCTION}

Now we show how the mountain pass lemma (Theorem 11) can be used to prove the theorems of $\S 2$. First we give the

Proof of Theorem 6. We apply Theorem 11 to $G(u)$ given by (2.6). We take $\mathscr{H}=D$ with norm

$$
\|u\|\left\|:=\left[a(u)+K\|u\|^{2}\right]^{1 / 2} \geq\right\| u \|,
$$

where $K$ is sufficiently large. We must show that $G$ has a continuous derivative. Let $u, h$ be any two functions in $D$. Then

$$
t^{-1} \int_{\Omega}[F(x, u+t h)-F(x, u)] d x=\int_{\Omega} \int_{0}^{1} f(x, u+\Theta t h) h d \Theta d x .
$$

The integrand converges a.e. to $f(x, u) h(x)$ as $t \rightarrow 0$. By hypothesis II, it is bounded in absolute value by

$$
\left(C V_{0} h\right)\left[V_{0}^{q-1}\left(|u|^{q-1}+|h|^{q-1}\right)+k_{1}\right] .
$$

Since $u, h$ are in $D$, the second factor is in $L^{q^{\prime}}(\Omega)$ while the first is in $L^{q}(\Omega)$. Thus the expression (4.3) is in $L^{1}(\Omega)$ and majorizes the integrand of (4.2). Hence the right-hand side of (4.2) converges to

$$
\int_{\Omega} f(x, u) h(x) d x \text {. }
$$

This shows that

$$
\left(G^{\prime}(u), h\right)=2 a(u, h)-\int_{\Omega} f(x, u) h d x .
$$

Next we check that (3.6) holds. If $u_{k}$ converges weakly to $u$ in $D$, then

$$
a\left(u_{k}-u, h\right) \rightarrow 0, \quad h \in D,
$$

and there is a subsequence for which $V_{0} u_{k}$ converges strongly in $L^{q}(\Omega)$ and another which converges a.e. But

$$
\left|f\left(x, u_{k}\right) h(x)\right| \leq\left|V_{0} u_{k}\right|^{q-1}\left|V_{0} h\right|+k_{1}\left|V_{0} h\right|,
$$

and $V_{0} u_{k}$ converges to $V_{0} u$ in $L^{q}(\Omega)$. Thus $\left|V_{0} u_{k}\right|^{q-1}$ converges to $\left|V_{0} u\right|^{q-1}$ in $L^{q^{\prime}}(\Omega)$, and consequently the right-hand side of (4.6) converges to $\left|V_{0} u\right|^{q-1}\left|V_{0} h\right|$ $+k_{1}\left|V_{0} h\right|$ in $L^{1}(\Omega)$. Hence

$$
\int_{\Omega} f\left(x, u_{k}\right) h(x) d x \rightarrow \int_{\Omega} f(x, u) h(x) d x .
$$


By (4.5) and (4.6)

$$
\left(G^{\prime}\left(u_{k}\right), h\right) \rightarrow\left(G^{\prime}(u), h\right) .
$$

Thus if $G^{\prime}\left(u_{k}\right) \rightarrow 0$, we must have $G^{\prime}(u)=0$.

Next we take $R_{0}$ so large that

$$
\max \left\{G(v),\|v\|=R_{0}, v \in N\right\}<c_{1} .
$$

This is possible by (2.5). Next, let $c$ be any number satisfying $c_{1} \leq c$, and let $c^{\prime}=\max \left(c_{1}, c-\sigma\right)$, where $0<\sigma<1$. Let $b=b\left(c^{\prime}\right)$, and take $\varepsilon<b+c$. If $G(u)$ satisfies $(3.10)$, then

$$
\frac{1}{2}\left(G^{\prime}(u), u\right) \geq c^{\prime}+\int_{\Omega} H(x, u) d x .
$$

Take $R$ so large that

$$
\int_{\Omega} H(x, u) d x+\frac{\Theta}{2}\left\|G^{\prime}(u)\right\|\|u\|>b-\varepsilon, \quad\|u\| \geq R,
$$

and $R \geq R_{0}$. Then for $u$ satisfying (3.10) we have

$$
\frac{1}{2}\left(G^{\prime}(u), u\right)+\frac{\Theta}{2}\left\|G^{\prime}(u)\right\|\|u\|>c^{\prime}+b-\varepsilon>0 .
$$

Thus (3.9) holds. Theorem 11 tells us that there is a $u \in D$ such that $G^{\prime}(u)=0$. This is precisely what we want in view of (4.4).

Proof of Theorem 7. We shall show that for any $\Theta>0$ and $\sigma<b_{0}+c_{1}$ there is an $R$ such that

$$
\left(G^{\prime}(u), u\right)+\Theta\left\|G^{\prime}(u)\right\|\|u\|>0
$$

holds for all $u$ satisfying (3.10). If this were not so, there would be a sequence $\left\{u_{k}\right\} \subset D$ such that

$$
\left\|u_{k}\right\| \rightarrow \infty, \quad\left\|G^{\prime}\left(u_{k}\right)\right\|\left\|u_{k}\right\| \leq C
$$

and

$$
\lim _{k \rightarrow \infty}\left(G^{\prime}\left(u_{k}\right), u_{k}\right) \leq 0
$$

(Recall that $\frac{1}{2}\left(G^{\prime}(u), u\right) \geq c-\sigma+b_{1}$ when (3.10) holds by (4.10) and (2.11).) Let $M^{\prime}=\left[N^{\prime} \oplus N(A)\right]^{\perp} \cap D$. We write $u_{k}=v_{0 k}+v_{k}+w_{k}$, where $v_{0 k} \in N(A)$, $v_{k} \in N^{\prime}$ and $w_{k} \in M^{\prime}$. In view of (4.14) we have

$$
\left|2 a\left(v_{k}\right)-\left(f\left(u_{k}\right), v_{k}\right)\right| \leq C, \quad\left|2 a\left(w_{k}\right)-\left(f\left(u_{k}\right), w_{k}\right)\right| \leq C .
$$

Now by (4.6), (4.1) and hypothesis II

$$
|(f(u), h)| \leq\left(\left\|V_{0} u\right\|_{q}^{q-1}+\left\|k_{1}\right\|_{q^{\prime}}\right)\|h\|_{q} \leq C_{1}\left(|\|u\||^{q-1}+1\right)\|\| h \| \mid .
$$

Thus if we put $t_{k}=\left\|\mid u_{k}\right\| \|,(4.16)$ implies

$$
\left|a\left(v_{k}\right)\right|+a\left(w_{k}\right) \leq C+C_{1}\left(t_{k}^{q}+t_{k}\right) .
$$

Let $\tilde{u}_{k}=u_{k} / t_{k}$. Then $\left\|\mid \tilde{u}_{k}\right\|=1$ and (4.17) implies

$$
\left\|\left|\tilde{v}_{k}+\tilde{w}_{k} \|\right| \rightarrow 0\right. \text {. }
$$


Hence

$$
\left\|\mid \tilde{v}_{0 k}\right\|=K\left\|\tilde{v}_{0 k}\right\| \rightarrow 1 \text {. }
$$

Thus there is a subsequence for which $\tilde{v}_{0 k} \rightarrow \tilde{v}_{0}, \tilde{v}_{k} \rightarrow 0, \tilde{w}_{k} \rightarrow 0$ in $L^{2}(\Omega)$ and a.e. Since $\tilde{v}_{0} \not \equiv 0, \tilde{v}_{0} \neq 0$ a.e., and

$$
\left|u_{k}\right| / t_{k}=\left|\tilde{v}_{0 k}+\tilde{v}_{k}+\tilde{w}_{k}\right| \rightarrow\left|\tilde{v}_{0}\right| \quad \text { a.e. }
$$

Hence $\left|u_{k}\right| \rightarrow \infty$ a.e. This implies

$$
\liminf _{k \rightarrow \infty} H\left(x, u_{k}\right) \geq W_{0}(x), \quad H\left(x, u_{k}\right) \geq W_{1}(x),
$$

and consequently

$$
\liminf _{k \rightarrow \infty}\left(G^{\prime}\left(u_{k}\right), u_{k}\right) \geq c-\sigma+b_{0}>0,
$$

contradicting (4.15). The result now follows from Theorem 11.

Proof of Proposition 1. If $w$ is in $M$,

$$
G(w) \geq \frac{1}{2} \lambda_{+}\|w\|^{2}-\int_{\Omega} F(x, w) d x \geq-b_{0} .
$$

Thus (2.4) holds with $c_{1} \geq-b_{0}$. On the other hand for $v \in N$

$$
G(v) \leq-\int_{\Omega} F(x, v) d x .
$$

But

$$
\liminf _{\|v\| \rightarrow \infty} \int_{\Omega} F(x, v) d x \geq b_{2} .
$$

For otherwise there would be a sequence $\left\{v_{k}\right\} \subset N$ and an $\varepsilon>0$ such that $\left\|v_{k}\right\| \rightarrow \infty$ and

$$
\int_{\Omega} F\left(x, v_{k}\right) d x \rightarrow b_{2}-\varepsilon .
$$

Let $t_{k}=\left\|v_{k}\right\|$ and $\tilde{v}_{k}=v_{k} / t_{k}$. Then $\left\|\tilde{v}_{k}\right\|=1$, and consequently there is a subsequence which converges to some $\tilde{v} \in N$. Thus $\tilde{v} \not \equiv 0$ and hence $\tilde{v} \neq 0$ a.e. This means that $\left|v_{k}\right|=t_{k}\left|\tilde{v}_{k}\right| \rightarrow \infty$ a.e. From this we see that

$$
\liminf \int_{\Omega} F\left(x, v_{k}\right) d x \geq b_{2},
$$

contradicting (4.19).

In proving Theorem 8 we shall make use of

Lemma 2. Suppose there are functions $W_{6}(x), W_{7}(x)$ in $L^{1}(\Omega)$ such that

$$
\liminf _{|t| \rightarrow \infty} M(x, t) \geq W_{6}(x), \quad M(x, t) \geq W_{7}(x) \text { a.e. }
$$

Then

$$
\liminf \int_{\Omega} M(x, u) d x \geq b_{6}:=\int_{\Omega} W_{6}(x) d x,
$$

as $\|u\| \rightarrow \infty$ provided $\hat{a}(u):=\frac{1}{2}(|A| u, u) \leq C$.

Before proving the lemma we show how it can be used in giving the 
Proof of Theorem 8. Let $R_{0}$ be chosen as in the proof of Theorem 6. For $u \in D$ we write $u=v_{0}+v+w$, where $v_{0} \in N(A), v \in N^{\prime}$ and $w \in M^{\prime}$. We note that

$$
\begin{aligned}
& |\Theta|\left(G^{\prime}(u), w-v+(\operatorname{sgn} \Theta) v_{0}\right) \\
& \quad=|\Theta|\left(2 a(w)-2 a(v)-(\operatorname{sgn} \Theta)\left(f, v_{0}\right)\right) \\
& \quad=|\Theta|(2 a(w)-2 a(v)-(\operatorname{sgn} \Theta)(f, u)+(\operatorname{sgn} \Theta)(f, v+w)) \\
& \quad=-\Theta(f, u)+|\Theta|(2 a(w)-2 a(v)+(\operatorname{sgn} \Theta)(f, v+w))
\end{aligned}
$$

Since

$$
\begin{gathered}
\|f\| \leq\left\|V_{1}\right\|, \\
K(u):=a(w)-a(v)+\frac{1}{2}(\operatorname{sgn} \Theta)(f, v+w) \rightarrow \infty,
\end{gathered}
$$

as $\hat{a}(u)=a(w)-a(v) \rightarrow \infty$. By (4.10) and (4.22)

$$
\begin{aligned}
\frac{1}{2}\left(G^{\prime}(u), u\right)+\frac{1}{2}|\Theta|\left\|G^{\prime}(u)\right\|\|u\| & \geq c-\sigma+\int_{\Omega} H_{\Theta}(x, u) d x+|\Theta| K(u) \\
& \geq c-\sigma+b_{5}+|\Theta| K(u) .
\end{aligned}
$$

We take $\sigma<b_{4}+c_{1}$ and take $C_{2}$ so large that the right-hand side of (4.24) and $K(u)$ are positive for $\hat{a}(u) \geq C_{2}$. This can be done by (4.23). We can now apply Lemma 2 to conclude that

$$
\liminf \int_{\Omega} H_{\Theta}(x, u) d x \geq b_{4},
$$

as $\|u\| \rightarrow \infty$ provided $\hat{a}(u) \leq C_{2}$. Thus we can take $R$ so large that the lefthand side of (4.24) is positive when $\|u\| \geq R$ and $\hat{a}(u) \leq C_{2}$. We see that the left-hand side of (4.24) is positive when $\|u\|=R$. The conclusion now follows from Theorem 11.

Proof of Lemma 2. We shall show that for each $c, \varepsilon>0$ there is an $R>0$ such that

$$
\int_{\Omega} M(x, u) d x>b_{6}-\varepsilon
$$

holds whenever $\hat{a}(u) \leq c$ and $\|u\|>R$. If (4.25) did not hold, there would be constants, $C, \varepsilon>0$ and a sequence $\left\{u_{k}\right\}$ such that $\hat{a}\left(u_{k}\right) \leq C,\left\|u_{k}\right\| \rightarrow \infty$ and

$$
\int_{\Omega} M\left(x, u_{k}\right) d x \leq b_{6}-\varepsilon \text { for each } k .
$$

We write $u_{k}=v_{0 k}+v_{k}+w_{k}$, where $v_{0 k} \in N(A), v_{k} \in N^{\prime}, w_{k} \in M^{\prime}$. By hypothesis II, there is a subsequence (also denoted $\left\{u_{k}\right\}$ ) such that $V_{0} v_{k} \rightarrow V_{0} v$, $V_{0} w_{k} \rightarrow V_{0} w$ in $L^{q}(\Omega)$, and we may assume that $v_{k} \rightarrow v, w_{k} \rightarrow w$ a.e. Thus $t_{k}=\left\|v_{0 k}\right\| \rightarrow \infty$. Put $v_{0 k}^{\prime}=v_{0 k} / t_{k}$. Then $\left\|v_{0 k}^{\prime}\right\|=1$. Since $\operatorname{dim} N(A)<\infty$, there is a subsequence (renamed) such that $v_{0 k}^{\prime} \rightarrow v_{0}^{\prime}$ in norm and a.e. Since $v_{0}^{\prime} \not \equiv 0$, we have $v_{0}^{\prime} \neq 0$ a.e. Consequently, $\left|v_{0 k}\right|=t_{k}\left|v_{0 k}^{\prime}\right| \rightarrow \infty$ a.e. Since $v$ and $w$ are finite a.e., we see that $\left|u_{k}\right| \rightarrow \infty$ a.e. Thus

$$
\liminf _{k \rightarrow \infty} M\left(x, u_{k}\right) \geq W_{6}(x), \quad M\left(x, u_{k}\right) \geq W_{7}(x), \quad \text { a.e. }
$$


This implies

contradicting (4.26).

$$
\liminf _{k \rightarrow \infty} \int_{\Omega} M\left(x, u_{k}\right) d x \geq b_{6}
$$

Proof of Theorem 9. For $w \in M$, write $w=w^{\prime}+v_{1}$, where $w^{\prime} \in M^{\prime}$ and $v_{1} \in N_{1}$. Then we have

$$
\begin{aligned}
G(w) & =a\left(w^{\prime}\right)-\int F\left(x, w^{\prime}+v_{1}\right) d x \geq \frac{1}{2} \lambda_{+}\left\|w^{\prime}\right\|^{2}-\left\|V_{1}\right\|\left\|w^{\prime}\right\|-\alpha_{1} \\
& \geq-\left(\left\|V_{1}\right\|^{2} / 2 \lambda_{+}\right)-\alpha_{1} .
\end{aligned}
$$

This gives (2.4). For $v \in N$, we write $v=v^{\prime}+v_{0}, v^{\prime} \in N^{\prime}, v_{0} \in N_{0}$. Then

$$
\begin{aligned}
G(v) & =a\left(v^{\prime}\right)-\int F\left(x, v^{\prime}+v_{0}\right) d x \\
& \leq a\left(v^{\prime}\right)-\int F\left(x, v_{0}\right) d x+\left\|V_{1}\right\|\left\|v^{\prime}\right\| \\
& \leq \frac{1}{2} \lambda_{-}\left\|v^{\prime}\right\|^{2}+\left\|V_{1}\right\|\left\|v^{\prime}\right\|-\int F\left(x, v_{0}\right) d x,
\end{aligned}
$$

where $\lambda_{-}$is the largest negative point in $\sigma(A)$. By (2.22),

$$
K_{0}=\inf _{N_{0}} \int F\left(x, v_{0}\right) d x>-\infty .
$$

Thus

$$
G(v) \leq \frac{1}{2} \lambda_{-}\left\|v^{\prime}\right\|^{2}+\left\|V_{1}\right\|\left\|v^{\prime}\right\|-K_{0}
$$

On the other hand

$$
G(v) \leq\left(\left\|V_{1}\right\|^{2} / 2 \lambda_{-}\right)-\int F\left(x, v_{0}\right) d x .
$$

Thus there is an $R$ such that $G(v)<c_{1}$ will hold if either $2\left\|v^{\prime}\right\| \geq R$ or $2\left\|v_{0}\right\| \geq R$. It will therefore hold if $\left\|v^{\prime}+v_{0}\right\| \geq R$. This proves (2.5).

Proof of Theorem 1. We apply Theorem 6. Hypothesis I is satisfied with $A=$ $-\Delta-\lambda_{l}$. We take $N_{0}=N(A)=E_{l}$ and note that by (1.5)

$$
G(w) \geq a(w)-\frac{1}{2} b\|w\|^{2}-B \geq-B, \quad w \in M
$$

Hence $c_{1} \geq-B$. Thus (2.4) holds. Next we note that if $u \equiv 0$ is not a solution of (1.12), then $c_{1}>-B$. To see this we make use of the formulas

$$
\begin{aligned}
F(x, t) & =\frac{1}{2} b_{+}(x) t^{2}+F_{0}(x, t), & & t>0, \\
& =\frac{1}{2} b_{-}(x) t^{2}+F_{0}(x, t), & & t<0 .
\end{aligned}
$$

$$
\begin{aligned}
F_{0}(x, t) & =2 t^{2} \int_{t}^{\infty} s^{-3} H(x, s) d s, & & t>0 \\
& =-2 t^{2} \int_{-\infty}^{t} s^{-3} H(x, s) d s, & & t<0
\end{aligned}
$$


These formulas are easily derived from the fact that

$$
\partial\left(F t^{-2}\right) / \partial t=-2 t^{-3} H(x, t) .
$$

Now suppose $c_{1}=-B$. Then there is a sequence $\left\{w_{k}\right\} \subset M$ such that $G\left(w_{k}\right) \rightarrow-B$. If the $a\left(w_{k}\right)$ are bounded, then there is a subsequence (renamed) which converges weakly in $D, w_{k} \rightarrow w$ in $L^{2}(\Omega)$ and a.e. in $\Omega$. Thus

$$
\begin{aligned}
\int_{\Omega} F\left(x, w_{k}\right) d x= & \frac{1}{2} \int b_{+}(x) w_{k+}(x)^{2} d x+\frac{1}{2} \int b_{-}(x) w_{k-}(x)^{2} d x \\
& +\int_{\Omega} F_{0}\left(x, w_{k}\right) d x \\
\rightarrow & \frac{1}{2} \int b_{+} w_{+}(x)^{2} d x+\frac{1}{2} \int b_{-} w_{-}(x)^{2} d x+\int_{\Omega} F_{0}(x, w) d x .
\end{aligned}
$$

Since $a(w) \leq \liminf a\left(w_{k}\right)$, we have $G(w)=c_{1}$. Thus

$$
a(w)+B \leq \int F(x, w) d x \leq \frac{1}{2} b\|w\|^{2}+B \leq a(w)+B .
$$

Thus $a(w)=\frac{1}{2} b\|w\|^{2}$, implying that $w \in E_{l+1}$. Also

$$
F(x, w) \equiv \frac{1}{2} b w^{2}+W(x) .
$$

In view of (1.5), this implies $[b-b(x)] w(x)^{2} \leq 0$. By hypothesis, $b(x) \leq b$ and $b(x) \not \equiv b$. The only way we can obtain $\leq 0$ is if $w(x)=0$ on a set of positive measure. But then $w(x) \equiv 0$. This implies $W(x) \equiv 0, F(x, t) \leq \frac{1}{2} b t^{2}$, $f(x, 0) \equiv 0$. On the other hand, if $t_{k}^{2}=a\left(w_{k}\right) \rightarrow \infty$, let $\tilde{w}_{k}=w_{k} / t_{k}$. Again there is a subsequence (renamed) such that $\tilde{w}_{k} \rightarrow \tilde{w}$ weakly in $D, \tilde{w}_{k} \rightarrow \tilde{w}$ in $L^{2}(\Omega)$ and a.e. in $\Omega$. Thus

$$
\int b_{+} \tilde{w}_{k+}^{2}+\int b_{-} \tilde{w}_{k-}^{2} \rightarrow \int b_{+} \tilde{w}_{+}^{2}+\tilde{b}_{-} \tilde{w}_{-}^{2} \leq b\|\tilde{w}\|^{2} \leq 2 .
$$

If this limit does not equal 2 , then

$$
G\left(w_{k}\right)=t_{k}^{2}\left[1-\frac{1}{2} \int b_{+} \tilde{w}_{k+}^{2}-\frac{1}{2} \int b_{-} \tilde{w}_{k-}^{2}\right]-\int F_{0}\left(x, t_{k} \tilde{w}_{k}\right) d x
$$

will converge to $\infty$ as $k \rightarrow \infty$. Thus we must have equality in (4.29). In particular, we see that $\tilde{w} \not \equiv 0$. Thus $\tilde{w} \neq 0$ a.e. (the unique continuation property). Consequently

$$
b_{+}(x) \equiv b \quad \text { when } \tilde{w}>0, \quad b_{-}(x) \equiv b \quad \text { when } \tilde{w}<0 .
$$

Since by (1.15)

we have

$$
\frac{1}{2} b_{+} w_{k}^{2}+F_{0}\left(x, w_{k}\right) \leq \frac{1}{2} b w_{k}^{2}+W(x),
$$

$$
F_{0}\left(x, w_{k}\right) \leq W(x) \text { when } w_{k}>0, \tilde{w}>0 .
$$

Consequently $H_{+}(x) \leq W(x)$ when $\tilde{w}>0$. Similar reasoning gives $H_{-}(x) \leq$ $W(x)$ when $\tilde{w}<0$. But

$$
-B \leftarrow G\left(w_{k}\right) \geq-\int F_{0}\left(x, t_{k} \tilde{w}_{k}\right) d x \rightarrow-\int_{\tilde{w}>0} H_{+}(x) d x-\int_{\tilde{w}<0} H_{-}(x) d x .
$$


Thus

This means that

$$
B \leq \int_{\tilde{w}>0} H_{+}(x) d x+\int_{\tilde{w}<0} H_{-}(x) d x \leq B
$$

$$
H_{+}(x) \equiv W(x) \quad \text { when } \tilde{w}>0, \quad H_{-}(x) \equiv W(x) \quad \text { when } \tilde{w}<0 .
$$

But (4.30) and (4.31) contradict the hypotheses of the theorem. Thus $c_{1}>-B$.

Next we show that $c_{0} \leq-B$. Let $\left\{v_{k}\right\} \subset N$ be such that $G\left(v_{k}\right) \rightarrow c_{0}$, $t_{k}=\left\|v_{k}\right\| \rightarrow \infty$. Let $\tilde{v}_{k}=v_{k} / t_{k}$. Then $\left\|\tilde{v}_{k}\right\|=1$, and consequently there is a subsequence (renamed) such that $\tilde{v}_{k} \rightarrow \tilde{v}$ in $N$. Since

$$
G\left(v_{k}\right)=t_{k}^{2}\left[a\left(\tilde{v}_{k}\right)-\frac{1}{2} \int_{\Omega} b_{+} \tilde{v}_{k+}^{2} d x-\frac{1}{2} \int_{\Omega} b_{-} \tilde{v}_{k-}^{2} d x\right]-\int_{\Omega} F_{0}\left(x, t_{k} \tilde{v}_{k}\right) d x
$$

and $a\left(\tilde{v}_{k}\right) \rightarrow a(\tilde{v})$, we will have $G\left(v_{k}\right) \rightarrow-\infty$ unless

$$
a(\tilde{v})=0, \quad b_{+} \tilde{v}_{+}^{2} \equiv 0, \quad b_{-} \tilde{v}_{-}^{2} \equiv 0 .
$$

This implies $\tilde{v} \in E_{l}$, and it is a solution of (1.10). By unique continuation $\tilde{v} \neq 0$ a.e. Thus $\left|v_{k}\right| \rightarrow \infty$ a.e., and we have

$$
\begin{aligned}
\lim \sup G\left(v_{k}\right) & \leq-\liminf \int_{\Omega} F_{0}\left(x, t_{k} \tilde{v}_{k}\right) d x \\
& =-\int_{\tilde{v}>0} H_{+}(x) d x-\int_{\tilde{v}<0} H_{-}(x) d x \leq-B,
\end{aligned}
$$

by hypothesis. Thus (2.5) holds. Finally we verify (2.8). An examination of (4.27) (4.28) shows that

$$
|f(x, t)| \leq C\left(|t|+W_{1}(x)|t|^{-1}\right),
$$

and

$$
f(x, t) / t \rightarrow b_{ \pm}(x) \quad \text { as } t \rightarrow \pm \infty \quad \text { a.e. }
$$

Thus $f(x, t)$ satisfies hypothesis I with $q=2$. Let $\left\{u_{k}\right\}$ be a sequence such that (4.14) holds. Let $t_{k}=\left\|u_{k}\right\|$ and $\tilde{u}_{k}=u_{k} / t_{k}$. Then (4.16) holds. By (4.32) we have

$$
a\left(w_{k}\right) \leq C\left(\left\|u_{k}\right\|\left\|w_{k}\right\|+1\right), \quad\left|a\left(v_{k}\right)\right| \leq C\left(\left\|u_{k}\right\|\left\|v_{k}\right\|+1\right) .
$$

This shows that $\left\|\tilde{u}_{k}\right\| \| \leq C$. Since the embedding of $D$ into $L^{2}(\Omega)$ is compact, there is a subsequence (renamed) such that $\tilde{u}_{k} \rightarrow \tilde{u}$ weakly in $D$, strongly in $L^{2}(\Omega)$ and a.e. in $\Omega$. Since

$$
\left|a\left(\tilde{u}_{k}, h\right)-\frac{1}{2} t_{k}^{-1}\left(f\left(x, u_{k}\right), h\right)\right| \leq C\|h\| / t_{k} \quad \forall h \in D,
$$

we have in the limit

$$
a(\tilde{u}, h)=\frac{1}{2} \int\left(b_{+} \tilde{u}_{+}-b_{-} \tilde{u}_{-}\right) h d x, \quad h \in D .
$$

Thus $\tilde{u}$ is a solution of

$$
A u=b_{+} u_{+}-b_{-} u_{-} \quad \text { in } \Omega, \quad u=0 \text { on } \partial \Omega .
$$

By the unique continuation property, $\tilde{u}$ cannot vanish on a set of positive measure since $\|\tilde{u}\|=1$. Thus $\left|u_{k}\right|=t_{k}\left|\tilde{u}_{k}\right| \rightarrow \infty$ a.e., as $k \rightarrow \infty$. 
Now suppose $\left\{u_{k}\right\}$ is a sequence satisfying $G\left(u_{k}\right) \geq c_{1},\left\|u_{k}\right\| \rightarrow \infty$. Then we claim that for any $\Theta>0$

$$
\liminf _{k \rightarrow \infty}\left[\left(G^{\prime}\left(u_{k}\right), u_{k}\right)+\Theta\left\|G^{\prime}\left(u_{k}\right)\right\|\left\|u_{k}\right\|\right]>0 .
$$

For

$$
\frac{1}{2}\left(G^{\prime}\left(u_{k}\right), u_{k}\right)=G\left(u_{k}\right)+\int H\left(x, u_{k}\right) d x,
$$

is bounded from below. The only way (4.34) can fail to hold is if (4.14) holds. But then $\left|u_{k}\right| \rightarrow \infty$ a.e. In this case

$$
\int H\left(x, u_{k}\right) d x \rightarrow \int_{\tilde{u}>0} H_{+}(x) d x+\int_{\tilde{u}<0} H_{-}(x) d x .
$$

Hence

$$
\liminf \frac{1}{2}\left(G^{\prime}\left(u_{k}\right), u_{k}\right) \geq c_{1}+B>0 .
$$

Remark. Note that if there exists a $w \in E_{l+1} \backslash\{0\}$ satisfying (1.11), then

$$
G(t w)=-\int_{\Omega} F_{0}(x, t w) d x \rightarrow-B \quad \text { as } t \rightarrow \infty .
$$

In this case we have $c_{1}=-B$. Instead of assuming $b(x) \not \equiv b$ we can assume that there does not exist a $w \in E_{l+1} \backslash\{0\}$ such that

$$
F(x, w) \equiv \frac{1}{2} b w^{2}+W(x) .
$$

If we insist on a strict inequality in (1.9) we can dispense with the assumptions $b(x) \not \equiv b$ and the nonexistence of a $w \in E_{l+1} \backslash\{0\}$ satisfying (1.11).

Proof of Theorem 2. Clearly hypotheses I and II of Theorem 6 are satisfied. Note also that

$$
|F(x, t)| \leq q^{-1} C|t|^{q}+k_{1}|t| .
$$

Assume first that (1.16) holds. We take $A=\Delta-\lambda_{l}, N_{0}=N(A)=E_{l}, b=$ $\lambda_{l+1}-\lambda_{l}$. Thus

$$
q G(w) \geq \int_{\Omega}\left(\frac{q}{2} b w^{2}-C|w|^{q}-q k_{1}|w|\right) d x, \quad w \in M .
$$

Thus $G(w) \rightarrow \infty$ as $\|w\| \rightarrow \infty, w \in M$. This shows that (2.4) holds. On the other hand

$$
\begin{gathered}
q|t|^{-q} F(x, t) \rightarrow \pm h_{ \pm}(x) \quad \text { a.e., } \quad \text { as } t \rightarrow \pm \infty, \\
q|t|^{-q} H(x, t) \rightarrow \pm(1-q / 2) h_{ \pm}(x) \quad \text { a.e., } \quad \text { as } t \rightarrow \pm \infty,
\end{gathered}
$$

by (1.13). Let $\left\{v_{k}\right\} \subset N$ be such that $t_{k}=\left\|v_{k}\right\| \rightarrow \infty$. Let $\tilde{v}_{k}=v_{k} / t_{k}$. Then $\left\|\tilde{v}_{k}\right\|=1$ and there is a renamed subsequence such that $\tilde{v}_{k} \rightarrow \tilde{v}$ as $k \rightarrow \infty$. If $a\left(\tilde{v}_{k}\right) \leq-c<0$, then

$$
G\left(v_{k}\right)=t_{k}^{2}\left[a\left(\tilde{v}_{k}\right)-t_{k}^{-2} \int F\left(x, v_{k}\right) d x\right] \rightarrow-\infty .
$$


If $a\left(\tilde{v}_{k}\right) \rightarrow 0$, then $a(\tilde{v})=0$ and $\tilde{v} \in E_{l}$. Since $\tilde{v} \not \equiv 0, \tilde{v} \neq 0$ a.e., and $\left|v_{k}(x)\right|=t_{k}\left|\tilde{v}_{k}(x)\right| \rightarrow \infty$ a.e. But by (4.36)

$$
\begin{aligned}
q t_{k}^{-q} \int F\left(x, v_{k}\right) d x & =q \int\left[F\left(x, t_{k} \tilde{v}_{k}\right) / t_{k}^{q}\left|\tilde{v}_{k}\right|^{q}\right]\left|\tilde{v}_{k}\right|^{q} d x \\
& \rightarrow B(\tilde{v}) \quad \text { as } k \rightarrow \infty
\end{aligned}
$$

Thus

$$
\int F\left(x, v_{k}\right) d x \rightarrow \infty \text { as } k \rightarrow \infty
$$

by $(1.16)$. Hence

$$
G\left(v_{k}\right) \leq-\int F\left(x, v_{k}\right) d x \rightarrow-\infty \quad \text { as } k \rightarrow \infty .
$$

This shows that $c_{0}=-\infty$ and (2.5) is satisfied. Finally, suppose $\left\{u_{k}\right\}$ is a sequence such that

$$
\left|G\left(u_{k}\right)\right| \leq C, \quad t_{k}=\left\|u_{k}\right\| \rightarrow \infty .
$$

The only way (4.34) can fail to hold is if (4.14) holds. But this implies $\left|u_{k}(x)\right| \rightarrow$ $\infty$ a.e. (see the proof of Theorem 7). But then (4.37) implies

$$
q t_{k}^{-q} \int H\left(x, u_{k}\right) d x \rightarrow(1-q / 2) B(\tilde{u}) \text { as } k \rightarrow \infty,
$$

(see (4.38)). Hence

$$
\int H\left(x, u_{k}\right) d x \rightarrow \infty \text { as } k \rightarrow \infty .
$$

If we now make use of (4.35) and (4.38), we see that

$$
\left(G^{\prime}\left(u_{k}\right), u_{k}\right) \rightarrow \infty \text { as } k \rightarrow \infty,
$$

and the proof for this case is complete. If (1.17) holds, we take $N_{0}=\{0\}$. Thus $E_{l} \subset M$. Let $\left\{w_{k}\right\}$ be a sequence in $M$ such that $t_{k}=\left\|w_{k}\right\| \rightarrow \infty$. If $a\left(\tilde{w}_{k}\right) \geq c>0$, then

$$
G\left(w_{k}\right)=t_{k}^{2}\left[a\left(\tilde{w}_{k}\right)-t_{k}^{-2} \int F\left(x, w_{k}\right) d x\right] \rightarrow \infty .
$$

Otherwise $a\left(\tilde{w}_{k}\right) \rightarrow 0$. There is a $\tilde{w} \in M$ such that $\tilde{w}_{k} \rightarrow \tilde{w}$ weakly in $D$, strongly in $L^{2}(\Omega)$ and a.e. in $\Omega$. Thus $\tilde{w} \in E_{l}$. Since $\|\tilde{w}\|=1, \tilde{w} \neq 0$ a.e. Consequently, $\left|w_{k}(x)\right| \rightarrow \infty$ a.e. In view of (4.36)

$$
\begin{aligned}
q\left|t_{k}\right|^{-q} & \int F\left(x, w_{k}\right) d x \\
& =q \int\left[F\left(x, t_{k} \tilde{w}_{k}\right) / t_{k}^{q}\left|\tilde{w}_{k}\right|^{q}\right]\left|\tilde{w}_{k}\right|^{q} d x \rightarrow B(\tilde{w}) \text { as } k \rightarrow \infty .
\end{aligned}
$$

Consequently

$$
G\left(w_{k}\right) \geq-\int F\left(x, w_{k}\right) d x \rightarrow \infty \quad \text { as } k \rightarrow \infty,
$$

and (2.4) holds. On the other hand, if $\left\{v_{k}\right\} \subset N$ and $t_{k}=\left\|v_{k}\right\| \rightarrow \infty$, then $a\left(\tilde{v}_{k}\right) \leq-c<0$ and

$$
G\left(v_{k}\right)=t_{k}^{2}\left[a\left(\tilde{v}_{k}\right)-t_{k}^{-2} \int F\left(x, v_{k}\right) d x\right] \rightarrow-\infty,
$$


as $k \rightarrow \infty$. Thus (2.5) holds. Finally, if the sequence $\left\{u_{k}\right\}$ satisfies (4.38) and (4.14) holds, then $\left|u_{k}(x)\right| \rightarrow \infty$ a.e., as before, and (4.37) implies

$$
\int H\left(x, u_{k}\right) d x \rightarrow-\infty \text { as } k \rightarrow \infty .
$$

It then follows from (4.35) and (4.38) that

$$
\left|\left(G^{\prime}\left(u_{k}\right), u_{k}\right)\right| \rightarrow \infty,
$$

contradicting (4.14). Thus (4.38) implies

$$
\left\|G^{\prime}\left(u_{k}\right)\right\|\left\|u_{k}\right\| \rightarrow \infty
$$

and the result follows.

Proof of Remark 1. Assumption (1.15) says that for each $v \in E_{l} \backslash\{0\}$ we have either

$$
\text { (a) }-\frac{B(v)}{B(-v)}<0, \quad \text { or } \quad\left(\text { b) }-\frac{B(v)}{B(-v)}>1\right. \text {. }
$$

If $B(v)>0$, this implies either

$$
\begin{aligned}
& \text { (a) } B(-v)>0, \quad \text { or } \\
& \text { (b) } B(-v)<0,
\end{aligned}
$$

If $B(v)<0,(4.39)$ implies either

(a) $B(-v)<0$, or

(b) $B(-v)>0$, and $-B(v)>B(-v)$.

In particular, if $B(v) B(-v)<0$, then we must have $|B(v)|>|B(-v)|$. By symmetry, we must also have $|B(-v)|>|B(v)|$, which is a contradiction. Thus only option (a) is possible.

Proof of Theorem 3. We apply Theorems 6 and 8. Hypotheses I and II are clearly satisfied. In view of (1.18) we see that $c_{1} \geq-B$. If $\left\{v_{k}\right\} \subset N$ and $t_{k}=\left\|v_{k}\right\| \rightarrow \infty$, let $\tilde{v}_{k}=v_{k} / t_{k}$. Then $\left\|\tilde{v}_{k}\right\|=1$ and there is a renamed subsequence such that $\tilde{v}_{k} \rightarrow \tilde{v}$ in $N$. If $a(\tilde{v}) \neq 0$, then

$$
G\left(v_{k}\right)=t_{k}^{2}\left[a\left(\tilde{v}_{k}\right)-t_{k}^{-2} \int F\left(x, v_{k}\right) d x\right] \rightarrow-\infty .
$$

If $a(\tilde{v})=0, \tilde{v} \in E_{l} \backslash\{0\}$. Hence $\tilde{v}(x) \neq 0$ a.e. Thus $\left|v_{k}(x)\right| \rightarrow \infty$ a.e., and

$$
G\left(v_{k}\right) \leq-\int F\left(x, v_{k}\right) d x \rightarrow-\int_{\tilde{v}>0} F_{+}(x) d x-\int_{\tilde{v}<0} F_{-}(x) d x<-B,
$$

by (1.21). Let $\varepsilon>0$ be so small that

$$
2 \varepsilon b_{2}<\int_{v>0} F_{+}(x) d x+\int_{v<0} f_{-}(x) d x-B, \quad v \in E_{l} \backslash\{0\},
$$

where $b_{2}:=\int W_{2}(x) d x$. If $\left\{u_{k}\right\}$ is a sequence satisfying (4.14), we have shown that $\left|u_{k}(x)\right| \rightarrow \infty$ a.e. If $\Theta<1$ is such that $1-\Theta<2 \varepsilon$, then

$$
H_{\Theta}=F-\frac{1}{2}(1-\Theta) t f \geq F-\varepsilon W_{2} .
$$


Thus

$$
\liminf _{t \rightarrow \pm \infty} H_{\Theta} \geq F_{ \pm}(x)-\varepsilon W_{2}
$$

and

$$
\liminf \int H_{\Theta}\left(x, u_{k}\right) d x \geq B+\varepsilon b_{2} .
$$

We can now apply Theorem 8 to conclude that hypothesis IV is satisfied.

Proof of Theorem 5. We apply Theorem 6 taking $q=2, A=-\Delta-\lambda_{l}$, and

$$
F(x, t)=\frac{1}{2} f_{+}(x) t_{+}^{2}+\frac{1}{2} f_{-}(x) t_{-}^{2}+W(x) \psi(t) .
$$

By (1.27), for $w \perp N$

$$
G(w) \geq-\int W(x) \psi(w) d x>-\|W\|_{1}=-B .
$$

Thus $c_{1}>-B$. On the other hand we claim

$$
G(v) \rightarrow-\infty \quad \text { as }\|v\| \rightarrow \infty, \quad v \in N .
$$

To see this let $\left\{v_{k}\right\} \subseteq N$ be a sequence such that $t_{k}=\left\{v_{k}\right\} \rightarrow \infty$. Let $\tilde{v}_{k}=v_{k} / t_{k}$. Then $\left\|\tilde{v}_{k}\right\|=1$ and there is a subsequence (renamed) for which $\tilde{v}_{k} \rightarrow \tilde{v}$ in $N$. Thus

$$
\begin{aligned}
t_{k}^{-2} G\left(v_{k}\right) & =a\left(\tilde{v}_{k}\right)-\frac{1}{2} \int\left(f_{+} \tilde{v}_{k+}^{2}+f_{-} \tilde{v}_{k-}^{2}\right) d x-t_{k}^{-2} \int W(x) \psi\left(v_{k}\right) d x \\
& \rightarrow a(\tilde{v})-\frac{1}{2} \int\left(f_{+} \tilde{v}_{+}^{2}+f_{-} \tilde{v}_{-}^{2}\right) d x<0
\end{aligned}
$$

by (1.26) since $\tilde{v} \not \equiv 0$. Thus $G\left(v_{k}\right) \rightarrow-\infty$ and (4.42) holds. Hence hypothesis III is verified. We now turn to (2.8). Let $\left\{u_{k}\right\}$ be a sequence such that (4.14) holds. Let $\tilde{u}_{k}=u_{k} / t_{k}$ where $t_{k}=\left\|u_{k}\right\|$. Then (4.16) holds. Since

$$
\begin{gathered}
|f(x, t)| \leq C(|t|+1), \quad a\left(w_{k}\right) \leq C\left(\left\|u_{k}\right\|\left\|w_{k}\right\|+1\right), \\
\left|a\left(v_{k}\right)\right| \leq C\left(\left\|u_{k}\right\|\left\|v_{k}\right\|+1\right) .
\end{gathered}
$$

This shows that $\left\|\tilde{u}_{k}\right\| \mid \leq C$. Since the embedding of $H_{0}^{\prime}(\Omega)=D\left(|A|^{1 / 2}\right)$ into $L^{2}(\Omega)$ is compact, there is a subsequence (renamed) such that $\tilde{u}_{k} \rightarrow \tilde{u}$ weakly in $H_{0}^{\prime}(\Omega)$, strongly in $L^{2}(\Omega)$ and a.e. in $\Omega$. Since

$$
\left|a\left(\tilde{u}_{k}, h\right)-\frac{1}{2} t_{k}^{-1}\left(f\left(x, u_{k}\right), h\right)\right| \leq C\|h\| / t_{k} \quad \forall h
$$

for each $k$, we have in the limit

$$
a(\tilde{u}, h)=\frac{1}{2} \int\left[f_{+} \tilde{u}_{+}-f_{-} \tilde{u}_{-}\right] h d x \quad \forall h .
$$

Hence $\tilde{u}$ is a solution of (4.33). By the unique continuation property $\tilde{u} \neq 0$ a.e., since $\|\tilde{u}\|=1$. Consequently, $\left|u_{k}\right|=t_{k}\left|\tilde{u}_{k}\right| \rightarrow \infty$ a.e.

Now suppose $\left\{u_{k}\right\}$ is a sequence such that

$$
G\left(u_{k}\right) \geq c_{1}, \quad\left\|u_{k}\right\| \rightarrow \infty .
$$

Then we claim that for any $\Theta>0$

$$
\liminf _{k \rightarrow \infty}\left[\left(G^{\prime}\left(u_{k}\right), u_{k}\right)+\Theta\left\|G^{\prime}\left(u_{k}\right)\right\|\left\|u_{k}\right\|\right]>0
$$


For

$$
\frac{1}{2}\left(G^{\prime}\left(u_{k}\right), u_{k}\right)=G\left(u_{k}\right)+\int W(x) \varphi\left(u_{k}\right) d x
$$

which is bounded from below. Thus the only way (4.43) can fail to hold is if (4.14) holds. But then we showed that $\left|u_{k}\right| \rightarrow \infty$ a.e. In this case by (1.24)

$$
\liminf _{k \rightarrow \infty} \frac{1}{2}\left(G^{\prime}\left(u_{k}\right), u_{k}\right) \geq c_{1}+B
$$

Proof of Theorem 4. This is a special case of Theorem 5. We have $N_{0}=E_{l}$, $N=N^{\prime} \oplus E_{l}$. Inequality (1.27) clearly holds since the left-hand side is $\geq$ $\left(\lambda_{l+1}-\lambda_{l}\right)\|w\|^{2}$ while the right-hand side is bounded above by this quantity. Inequality (1.26) also holds. For the right-hand side is never negative. If $v \in N$ is not in $E_{l}$, then the left-hand side of (1.26) is negative. Thus (1.26) holds for such $v$. On the other hand, if $v \not \equiv 0$ is in $E_{l}$, then the left-hand side vanishes and the right-hand side is positive by (1.25). Thus all of the hypotheses of Theorem 5 are satisfied.

\section{BIBLIOGRAPHY}

1. J. T. Schwartz, Generalizing the Lusternik-Schnirelmann theory of critical points, Comm. Pure Appl. Math. 17 (1964), 307-315.

2. R. S. Palais, Lusternik-Schnirelmann theory on Banach manifolds, Topology 5 (1966), 115132.

3. __ Critical point theory and the minimax principle, Proc. Sympos. Pure Math., vol. 15, Amer. Math. Soc., Providence, R. I., 1970, pp. 185-212.

4. A. Ambrosetti and P. H. Rabinowitz, Dual variational methods in critical point theory and applications, J. Funct. Anal. 14 (1973), 349-381.

5. D. C. Clark, $A$ variant of the Lusternik-Schnirelmann theory, Indiana Univ. Math. J. 22 (1972), 65-74.

6. L. Nirenberg, Variational and topological methods in nonlinear problems, Bull. Amer. Math. Soc. 4 (1981), 267-302.

7. P. Bartolo, V. Benci, and D. Fortunato, Abstract critical point theorems and applications to some nonlinear problems with "strong" resonance at infinity, Nonlinear Anal. Theory Methods Appl. 7 (1983), 981-1012.

8. P. H. Rabinowitz, Some minimax theorems and applications to nonlinear partial differential equations, Nonlinear Analysis (L. Cesari, R. Kannan, H. Weinberger, eds.), Academic Press, New York, 1978, pp. 161-177.

9. G. Cerami, Un criterio di esistenza per $i$ punti critici su varieta ellimitate, Rend. Istit. Lombardo Accad. Sci. Lett. 112 (1978), 332-336.

10. K. Thews, Nontrivial solutions of elliptic equations at resonance, Proc. Roy. Soc. Edinburgh 85A (1980), 119-129.

11. S. Ahmed, C. Lazer, and J. L. Paul, Elementary critical point theory and perturbations of elliptic boundary value problems at resonance, Indiana Univ. Math. J. 25 (1976), 933-944.

12. E. A. Landesman and A. C. Lazer, Nonlinear perturbations of linear elliptic boundary value problems at resonance, J. Math. Mech. 19 (1970), 609-623.

13. M. S. Berger and M. Schechter, On the solvability of semilinear gradient operator equations, Adv. in Math. 25 (1977), 97-132.

14. D. G. De Figueiredo, Some remarks on the Dirichlet problem for elliptic equations, Trab. Mathematica (Univ. de Brasilia) No. 57 (1974).

15. R. E. Gaines and J. L. Mawhin, Coincidence degree and nonlinear differential equations, (A. Dold and B. Eckmann, eds.), Lecture Notes in Math., vol. 568, Springer-Verlag, Berlin and New York, 1977. 
16. E. Tarafdar, An approach to nonlinear elliptic boundary value problems, J. Austral. Math. Soc. 34 (1983), 316-335.

17. P. H. Rabinowitz, Minimax methods in critical point theory with applications to differential equations, CBMS Regional Conf. Ser. in Math., no. 65, Amer. Math. Soc., Providence, R. I., 1986.

18. M. Schechter, The Hampwile theorem for nonlinear eigenvalues, Duke Math. J. 59 (1989), 325-335.

19. D. G. De Figueiredo, Positive solutions for some classes of semilinear elliptic problems, Proc. Sympos. Pure Math., vol. 45, Amer. Math. Soc., Providence, R. I., 1986, pp. 371-379.

20. P. Pucci and J. Serrin, A mountain pass theorem, J. Differential Equations 60 (1985), 142149.

21. Nguyen Phuong Cac, On an elliptic boundary value problem at double resonance, J. Math. Anal. Appl. 132 (1988), 473-483.

22. S. Ahmad, Multiple nontrivial solutions of resonant and nonresonant asymptotically linear problems, Proc. Amer. Math. Soc. 96 (1986), 405-409.

23. A. C. Lazer and P. J. McKenna, Multiplicity results for a semilinear boundary value problem with the nonlinearity crossing higher eigenvalues, Nonlinear Analysis Theory Methods Appl. 9 (1985), 335-349.

24. __ Critical point theory and boundary value problems with nonlinearities crossing multiple eigenvalues I, Comm. Partial Differential Equations 10 (1985), 107-150; II, Comm. Partial Differential Equations 11 (1986), 1653-1676.

25. S. Solimini, Some remarks on the number of solutions of some nonlinear elliptic equations, Ann. Inst. H. Poincaré Anal. Non Linéaire 2 (1985), 143-156.

26. A. Bahri and P. L. Lions, Remarks on variational critical point theory and applications, C. R. Acad. Sci. Paris 301 (1985), 145-147.

Department of Mathematics, University of California, Irvine, California 92717

E-mail address: scmrxp@uci.edu 